\title{
Variation in incidences of tuberculosis in subgroups of South African gold miners
}

\author{
Immo Kleinschmidt, Gavin Churchyard
}

\begin{abstract}
Objectives-To determine risk factors and to identify groups at high risk of tuberculosis (TB) among South African gold miners.

Methods-A retrospective cohort analysis was carried out with hospital and personnel databases on a random sample of the workforce of Freegold Mines, served by Ernest Oppenheimer Hospital, Welkom, South Africa. The outcome measure of interest was a reported first episode of TB while employed at Freegold. A personyears analysis was carried out to estimate incidence ratios between different categories of age group, period, cumulative service, mining occupation, and silicosis status. A separate analysis was carried out on a subgroup of men (non-random) whose HIV status was known.
\end{abstract}

Results-The main finding of the study was that TB was more strongly associated with age than expected with a rate ratio of reported incidence of 21 for the oldest age group compared with the youngest, after adjustment for period, cumulative service, occupation, and silicosis status. There was also a significant association between TB and occupations such as drilling with a rate ratio of 2.3 compared with low dust surface and maintenance workers, after adjustment for age, period, cumulative service, and silicosis. Analysis of the HIV tested subgroup showed that these results are unlikely to be the result of confounding due to HIV infection. Prevalence of HIV in this group has been rising sharply since 1991, but the increase was slowing towards the end of the study period.

Epidemiology Research Unit, PO Box 30606, Braamfontein 2017 South Africa I Kleinschmidt

Department of Medicine, Ernest Oppenheimer Hospital, PO Box 87, Welkom 9460, South Africa

G Churchyard

Correspondence to: Immo Kleinschmidt, Central Statistical Service, Private Bag X44, Pretoria 0001, South Africa.

A recent study on tuberculosis by the Department of Health has shown that South Africa faces possibly the world's worst epidemic of the disease, with a national incidence of $>3 / 1000 /$ year. ${ }^{1}$ In the mining industry, mine medical officers generally regard TB as one of the most serious health problems facing mineworkers, particularly in gold mining. ${ }^{2}$ Tuberculosis is the biggest single cause of mortality among mineworkers apart from trauma. ${ }^{3}$ The recent Commission of Inquiry into Safety and Health in the Mining Industry in South Africa (the Leon Commission) has expressed "grave concern over the failure to control TB in the mining industry," particularly in the light of the spread of HIV. ${ }^{4}$ There are no official statistics of TB among mineworkers, but there is evidence that there is considerable variation in incidences between mines ranging from $<10 / 1000 /$ year to $>20 / 1000 /$ year. $^{5}$

Unpublished data presented to the Leon Commission gave an indication of variation in TB incidences by occupation, with the highest incidence in those occupations with the highest exposure to dust, but it is not clear whether potential confounders such as age and silicosis had been taken into account. ${ }^{6}$

Silicosis has long been recognised as an important risk factor for tuberculosis. ${ }^{78}$ The question as to whether long term exposure to silica, without manifestation of silicosis, constitutes a risk factor for tuberculosis is unresolved. ${ }^{9}{ }^{10}$ A necropsy study of black mineworkers has shown that workers who had silicosis, evident at necropsy but not sufficiently progressed to be detected radiologically during life, had a significantly higher risk of tuberculosis than those without silicosis. ${ }^{11}$

Another more recent necropsy based study ${ }^{12}$ has concluded that increasing average age and increased duration of service are contributing towards a rise in the prevalence of TB in gold miners, but that there was nevertheless an underlying trend with time that could not be entirely explained by these factors. Studies based on routine necropsies have the benefit of reliable diagnosis, but are unable to measure incidence of disease and age of onset of disease and have not been able to take account of mining occupation. Given the competing potential risk factors of silicosis, age, duration of service, and mining occupation, as well as HIV infection, we analysed retrospective data over 21 years from 1975 to 1996 to estimate the relative contributions made by individual factors, to determine the variation in rates of TB in subgroups of mineworkers, and to identify groups that may be at particularly high risk.

\section{Methods and analysis}

The setting for this study is the workforce of Freegold mines, which are served by the Ernest Oppenheimer Hospital in Welkom, South 
Africa. Cases of tuberculosis at Freegold have been detected through passive presentation and a mass miniature radiograph screening programme. Diagnosis of tuberculosis was based on radiological, clinical, and bacteriological grounds with a weighted diagnostic protocol validated at the Ernest Oppenheimer Hospital. ${ }^{13}$ Diagnosis of tuberculosis includes pulmonary (smear positive and culture positive, smear negative and culture positive, and smear negative and culture negative), and extra-pulmonary forms. Patient data pertaining to all forms of tuberculosis at the hospital are captured on a central TB data base. Although it is possible that workers seek medical treatment elsewhere, this is highly unlikely during their mining years. For the purpose of this study, a random sample of 5000 company numbers was drawn from a list of 70000 company numbers obtained from the Occupational Health Centre at the hospital. The company numbers which constituted the sampling frame were those of all workers employed at Freegold mines who attended the Occupational Health Centre for a medical examination during 1994 or 1995. All workers attended an annual screening examination. Also a few attended before exit, leave, or at initial examinations. The records of men who had left the employment of Freegold before 1994 have been archived and could not easily be included in the sampling frame.

Personal data including date of birth, occupation, and starting and finishing dates for each contract worked, were retrieved for each of the 5000 men. The first occurrence of TB, if any, was obtained from the TB data base for the selected men. Diagnoses of silicosis were also known. These conform to a certification of category $\geqslant 1 / 1$ with the International Labour Office standard guidelines. ${ }^{14}$ These data thus provide work histories going back in time for all service done at Freegold for all participants in this study, as well as first episodes of TB and silicosis status for each worker. HIV status was not available for the main study, but it was known for a non-random subgroup of 1523 men who had had an HIV test for medical reasons at some time since 1991, when HIV testing was first introduced. A separate analysis of this subgroup was undertaken to see whether the results of the main study were likely to have been confounded by HIV status, and to make an assessment of the impact of HIV on incidence of TB.

Further work histories of the study group were obtained for any mining service before joining Freegold from the recruiting agency, the employment bureau of Africa. Most of the men could be traced in the employment bureau records, resulting in a modest additional contribution $(4.9 \%)$ to the total cumulative service.

The random sample of 5000 men in the study group collectively worked for a total of 30493 separate work contracts which constituted the observation time. The following data were attached to each employment contract record: employee identifier, age at start of contract, year of start of contract, total known pre- vious cumulative service in mining, mining occupation for the particular contract, whether the worker had previously been diagnosed with silicosis or not, duration of observation time for the particular contract, and whether a first episode of TB was recorded. If TB was recorded during the contract, observation time was ended on the date of diagnosis and all subsequent work records for the person were not used. If a worker was diagnosed as having silicosis during a work contract, the observation time was split into periods before and after silicosis, and all subsequent records for the worker were marked as after silicosis. In this way silicosis could be modelled as an exposure.

It was estimated that the study would have $90 \%$ power to detect an incidence rate ratio for TB of two for a group of men whose exposure was of particular interest-for example, older men, drillers who constituted at least $5 \%$ of the cohort. This was based on the assumption that the mean annual incidence of TB would be at least $0.5 \%$ and that the mean period of follow up would be 10 years per worker.

A person-years analysis ${ }^{15}$ was conducted to calculate incidence in various subgroups of workers, and to measure the size of several risk factors in a multivariate analysis. Observation time was expanded on three time scales: age group (10 year groups from 15 to 65), period (1975-83, 1984-9, and 1990-6), and cumulative service or time since start of exposure (two year periods up to 14 years service, one category for more than 14 years). Observation time was marked to be accumulated within particular occupational groups and whether or not silicosis had previously been diagnosed.

Data on dust exposure were not available but occupation was used as a surrogate measure. For this purpose several occupational groups were classified as follows: (a) a reference category of low exposure to dust was defined by grouping occupations that are not directly involved in production or development-for example, engineering maintenance, surface workers, caterers, store attendants, security patrolmen etc; $(b)$ occupations whose description implies underground work related to the production and removal of ore and rock. These included drillers, stope team workers, development team leaders, scraper winch operators, mining team, mining helpers, and miner's assistants; (c) an undifferentiated group of all other occupations combined.

For the main study, with data on the entire sample of men, crude (unadjusted) rates were calculated for age groups, periods, cumulative service categories, occupation groups, and silicosis status. To obtain rate ratios between exposure categories, corrected for confounders, the data were analysed with a multivariate Poisson regression model containing age, period, cumulative service, occupation, and silicosis as explanatory variables and the incidence of tuberculosis as the response variable. Interaction terms were added to the regression model to test whether any explanatory variable was modified by any one of the others. 
Table 1 Unadjusted tuberculosis incidence by age group, period, cumulative service, occupation, and silicosis status

\begin{tabular}{lcc}
\hline & Incidence/1000 person-years & $(95 \% C I)$ \\
\hline Age group: & & \\
15-24 & 1.10 & $(0.55$ to 2.21$)$ \\
$25-34$ & 5.53 & $(4.67$ to 6.54$)$ \\
$35-44$ & 11.47 & $(9.95$ to 13.23$)$ \\
$45-54$ & 17.12 & $(14.07$ to 20.82$)$ \\
$55-64$ & 30.95 & $(18.96$ to 50.51$)$ \\
Period: & & \\
1975-83 & 3.18 & $(2.25$ to 4.50$)$ \\
1984-9 & 7.27 & $(6.15$ to 8.60$)$ \\
1990-6 & 10.85 & $(9.65$ to 12.20$)$ \\
Cumulative service: & & \\
$0-1$ & 3.59 & $(2.55$ to 5.05$)$ \\
2-3 & 4.46 & $(3.27$ to 6.07$)$ \\
4-5 & 6.09 & $(4.66$ to 7.98$)$ \\
6-7 & 11.79 & $(9.63$ to 14.43$)$ \\
8-9 & 11.71 & $(9.38$ to 14.63$)$ \\
10-11 & 11.08 & $(8.50$ to 14.43$)$ \\
12-13 & 11.69 & $(8.61$ to 15.88$)$ \\
14 & 11.66 & $(8.95$ to 15.19$)$ \\
Occupation: & & \\
Low dust (surface and maintenance) & 4.75 & $(3.49$ to 6.48$)$ \\
Development team leader & 8.66 & $(3.61$ to 20.81$)$ \\
Driller & 15.98 & $(12.38$ to 20.63$)$ \\
Mining assistant & 6.64 & $(3.86$ to 11.43$)$ \\
Mining helper & 10.08 & $(7.05$ to 14.41$)$ \\
Mining team & 11.00 & $(8.90$ to 13.61$)$ \\
Stope team & 13.94 & $(9.96$ to 19.51$)$ \\
Winch operator & 8.67 & $(6.52$ to 11.54$)$ \\
Other & 6.33 & $(5.35$ to 7.49$)$ \\
Silicosis status: & & \\
No & 7.9 & $(7.19$ to 8.69$)$ \\
Yes & 26.39 & $(17.69$ to 39.37$)$ \\
\hline
\end{tabular}

Bivariate associations between some of the explanatory variables were investigated. Associations between silicosis and age and silicosis and cumulative service were analysed with a non-parametric test for trend. ${ }^{16} \mathrm{~A}$ simple $\chi^{2}$ test was performed to test for any association between silicosis and occupational group.

As the date of seroconversion in the subgroup infected with HIV could not be assessed, a cross sectional analysis was undertaken for the men tested for HIV, rather than the person-years cohort analysis used for the main study, which requires an estimate of exposure times for each risk factor. This meant that the incidence rate ratio for TB for the effect of HIV could not be calculated. Instead, the association between ever having had TB and current HIV status was estimated with the Mantel-Haenzel method, controlling for silicosis status, age, and cumulative service. Associations between HIV status and the other risk factors were also investigated.

\section{Results}

Of the 5000 men sampled, 24 were excluded as their work histories showed no period of service, either before or after their medical examination or because the company number was not allocated. Of the remaining 4976 men, 449 (9\%) had been diagnosed with a first episode of tuberculosis when in employment at Freegold. Total observation time amounted to 55822 person-years, giving a mean incidence of first episodes of TB over the 21 year period of 8.2 cases per 1000 person-years.

Table 1 summarises the unadjusted rates calculated for various subgroups, obtained from the main study comprising the entire sample. It shows the incidence for each 10 year age group rising from 1.1 in the 15 to 24 year age group to 31 for the 55 to 64 year age group.
The incidence for each of three consecutive periods, by which the follow up had been stratified, showed a secular rising trend in the incidence from 3.2 to 10.9 per 1000 personyears. The incidence for workers with different durations of previous cumulative service in mining rose from 3.6 for workers with over two years service, to $11.8 / 1000$ person-years for workers with seven to eight years previous exposure to mining. The rate remains relatively constant for workers with longer periods of previous service. Incidence by occupation ranged from 4.8 for surface and maintenance workers, to nearly $16 / 1000$ person-years for drillers. The unadjusted incidences for workers without diagnosed silicosis was 7.9 whereas that for silicotic patients was $26.4 / 1000$ person years, giving a crude relative risk of 3.3. Simple bivariate tests for association showed that silicosis is associated with age $(P<0.01)$, with cumulative service $(P<0.01)$, and with occupation $(\mathrm{P}=0.01)$.

Table 2 shows the results of the Poisson multivariate regression analysis, again for the main study of all men. Incidence rate ratios are given relative to baseline categories shown, and adjusted for the other variables in the model. The $P$ values show the results of likelihood ratio tests for each of the variables. After adjusting for period, cumulative service, occupation, and silicosis, the incidence rate ratio for the oldest age group (55 to 64 ) was still 21.2 compared with the youngest group (15 to 24 years). The incidence rate ratio for the period 1990-6 compared with the earlier period of 1975-83 was 1.8 . The rate ratio for cumulative service relative to the group with less than two years service was not significantly different from 1 for any number of years of service, once the data were adjusted for age, period, occupation, and silicosis status. Differences between occupational groups remained significant after adjustment for confounders, with rate ratios of $>2$ for drillers, stope teams, mining teams, and mining helpers compared with the low dust group.

The multivariate analysis was repeated assigning one of three categories of main occupation to each person, in place of the specific occupations. The results indicate that workers who have performed jobs that were mainly in the production group already defined, have a TB incidence rate ratio of 1.93 (95\% CI 1.43 2.7) compared with surface and maintenance workers, after adjustment for the other factors.

Interactions of silicosis with age, occupation, and cumulative service were tested and found to be non-significant.

The overall incidence of TB in the group tested for HIV was 16/1000 person-years. The figure shows since 1991 the rising prevalence of HIV infection in the subgroup of workers tested for HIV. Table 3 shows how HIV infection varies inversely with age $(P<0.01)$. There was a marginally significant inverse association between HIV status and cumulative service $(P=0.054)$, whereas the associations between HIV status and silicosis $(P=0.63)$ and HIV status and occupation $(\mathrm{P}=0.55)$ were both non-significant. Bivariate association between 
Table 2 Adjusted tuberculosis incidence rate ratios

\begin{tabular}{|c|c|c|c|}
\hline & Incidence rate ratio & $(95 \% C I)$ & Pvalue \\
\hline Age group: & & & $<0.0001$ \\
\hline $\begin{array}{l}15 \text { to } 24 \text { (reference) } \\
25-34 \\
35-44 \\
45-54 \\
55-64\end{array}$ & $\begin{array}{r}1 \\
3.99 \\
7.88 \\
10.86 \\
21.17\end{array}$ & $\begin{array}{l}(1.91 \text { to } 8.33) \\
(3.74 \text { to } 16.59) \\
(5.04 \text { to } 23.38) \\
(8.60 \text { to } 52.11)\end{array}$ & \\
\hline $\begin{array}{l}\text { Period: } \\
\text { 1975-83 (reference) } \\
1984-9 \\
1990-6\end{array}$ & $\begin{array}{l}1 \\
1.65 \\
1.83\end{array}$ & $\begin{array}{l}(1.09 \text { to } 2.50) \\
(1.19 \text { to } 2.82)\end{array}$ & 0.017 \\
\hline $\begin{array}{l}\text { Cumulative service: } \\
0-1 \text { (reference) } \\
2-3 \\
4-5 \\
6-7 \\
8-9 \\
10-11 \\
12-13 \\
\geqslant 14 \mathrm{~s}\end{array}$ & $\begin{array}{l}1 \\
0.86 \\
0.86 \\
1.39 \\
1.23 \\
1.04 \\
0.96 \\
0.83\end{array}$ & $\begin{array}{l}(0.54 \text { to } 1.38) \\
(0.54 \text { to } 1.37) \\
(0.89 \text { to } 2.17) \\
(0.77 \text { to } 1.95) \\
(0.64 \text { to } 1.72) \\
(0.57 \text { to } 1.63) \\
(0.49 \text { to } 1.39)\end{array}$ & 0.028 \\
\hline $\begin{array}{l}\text { Occupation: } \\
\text { Low dust (reference) } \\
\text { Development team leader } \\
\text { Driller } \\
\text { Mining assistant } \\
\text { Mining helper } \\
\text { Mining team } \\
\text { Stope team } \\
\text { Winch operator } \\
\text { Other }\end{array}$ & $\begin{array}{l}1 \\
1.33 \\
2.25 \\
1.36 \\
2.56 \\
2.15 \\
2.42 \\
1.54 \\
1.37\end{array}$ & $\begin{array}{l}(0.52 \text { to } 3.39) \\
(1.49 \text { to } 3.38) \\
(0.73 \text { to } 2.55) \\
(1.57 \text { to } 4.18) \\
(1.47 \text { to } 3.14) \\
(1.52 \text { to } 3.86) \\
(1.00 \text { to } 2.35) \\
(0.96 \text { to } 1.95)\end{array}$ & $<0.0001$ \\
\hline $\begin{array}{l}\text { Silicosis status: } \\
\text { No (reference) } \\
\text { Yes }\end{array}$ & $\begin{array}{l}1 \\
1.54\end{array}$ & (1.00 to 2.37 ) & 0.049 \\
\hline
\end{tabular}

Table 3 HIV infection by age group

\begin{tabular}{lllccc}
\hline \multicolumn{6}{l}{ Age group (y) } \\
\cline { 2 - 6 } & $15-24$ & $25-34$ & $35-44$ & $45-54$ & $55-64$ \\
\hline n & 36 & 613 & 592 & 230 & 52 \\
HIV positive (\%) & 19 & 17 & 12 & 9 & 10 \\
\hline
\end{tabular}

TB and HIV infection was non-significant $(P=0.16)$, but after controlling for age, $T B$ was significantly associated with being HIV positive (odds ratio $(\mathrm{OR}) 1.8, \mathrm{P}<0.01$ ). The adjusted OR for TB among workers infected with HIV relative to HIV seronegative workers was 1.6 $(\mathrm{P}=0.042)$ after controlling for age, cumulative service, occupation, and silicosis. Interactions between HIV and the other risk factors were non-significant in their effect on TB.

\section{Discussion}

Several known biases and shortcomings in the data affect the results of this study. These are:

(1) Selection bias. As already mentioned, the sample had to be drawn at the end of the follow up period, rather than at the beginning, so that

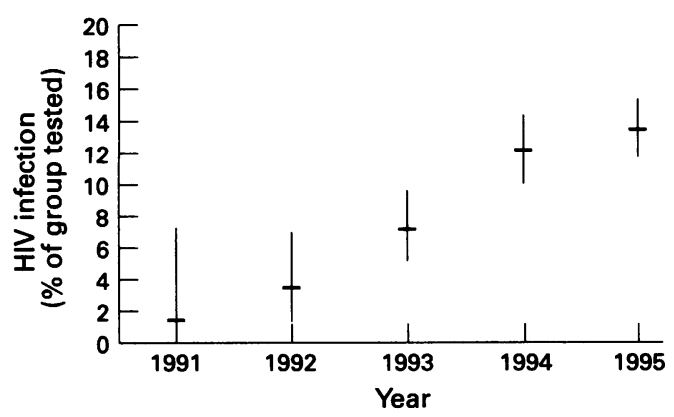

Figure 1 Prevalence of HIV infection (95\% confidence intervals) in the group tested for HIV. workers who left Freegold before 1994 were not included in the study. Some of these workers are likely to have left because of ill health, or may have died, so that the sample is biased towards the healthier workers, particularly for those with longer periods of service.

(2) Healthy worker effect. It is known that the healthier men self select themselves for physically demanding work such as mining, and recruitment is conditional upon a satisfactory medical examination. Once selected the healthiest are likely to be drawn to underground production jobs such as drilling, as these are better paid.

(3) Change in employment policy. Before 1984, miners with tuberculosis were repatriated ${ }^{17}{ }^{18}$ and would not have entered the sample unless they returned at some later stage. One would therefore expect the incidence to seem to be much lower before this date. Period has been divided with one break at 1983-4 to control for this change.

(4) The subgroup tested for HIV represents a high risk group of the workforce comprising a large proportion of patients with sexually transmitted diseases and TB.

Although these shortcomings in the data need to be taken into account when interpreting the results, they are unlikely to invalidate the main findings of this study as most of these factors will result in an underestimate of the true effect and thus in a bias towards unity.

The most striking result of this analysis is the steep gradient of incidence of TB with age, even after adjusting for known confounders. Department of Health figures for the South African population as a whole show that in 1993 the reported incidence of TB for black men was $2.4 / 1000$ for all age groups, rising from 1.6 for the 20 to 24 year old group to 5.5 for the 60 to 64 year old group. ${ }^{19}$ Although recognising that underreporting is likely to be a much bigger problem in the population at large than it is on the mines where active case finding is practised, comparison with the population nationally shows that the age effect measured in this population of gold miners is about five times as high as for comparable age groups of black men generally. ${ }^{20}$ The association between silicosis and age suggests that it is possible that the high incidence in older men may be in part due to residual confounding by undetected and undetectable silicosis in these men. Selection bias in favour of survivors will have acted against the association of TB with age. Recording of age itself may be subject to considerable error, due to many men not knowing their exact date of birth. This error is likely to be random, and would have diluted the association between TB and age. Therefore, apart from the possibility of residual confounding due to silicosis, biases in the data are likely to have had a negative effect on the rate ratio of older men compared with younger men and the true effect may well be even higher.

A possible explanation for a steep gradient of reported incidence of $T B$ with age may be an age related cohort effect due to older patients having been exposed to a higher prevalence of TB when they were young (B Fourie, personal 
communication). Another factor may be that both reactivation and exogenous reinfection occur more often in elderly people. This effect is, however, not specific to mineworkers and therefore does not explain why the incidence rate ratio with age should be so much higher than in the general population.

The small positive trend of incidence of TB with period after allowing for age, cumulative service, silicosis, and occupation, needs to be assessed against the positive biases introduced by selection bias and medical repatriation in the earlier period. The study therefore provides no conclusive evidence of an increase in incidence of TB that is independent of changes in mean age, prevalence of silicosis, and distribution of high risk occupations in the demography of the workforce.

The incidence of TB with previous cumulative service shows a positive trend, but this disappears once adjustment for other factors has been made. It is possible that any true association between service and incidence of TB has not been detected due to the bias in favour of long term survivors. It is also possible that the proxy measures of dust exposurenamely, occupation and cumulative servicehave led to considerable exposure misclassification, which would have resulted in a dilution of the effect of cumulative service.

The results show a significant association between the incidence of TB and occupation, even after correcting for known confounders. This result remains unaltered whether exposure is aggregated separately for each occupation, taking into account any changes in occupation by individual men, or whether each man is assigned an occupational group (main occupation) that he spent most of his previous service performing. The association with occupation has come about despite the potential for negation by the healthy worker effect. The only confounder which may have had a positive influence on the association with jobs that carry a higher burden of exposure to dust, is residual confounding due to silicosis, which in itself is the result of exposure to silica quartz. Our result thus confirms the unpublished data presented to the Leon Commission about this.

The results show a significant overall relative risk of TB of 3.3 for workers with silicosis, which is similar to what has been reported in other studies. Underascertainment of silicosis in workers without TB and late ascertainment of silicosis in workers with TB is likely to have blurred the strength of the association. The apparent absence of an effect due to silicosis after adjustment for the other factors may be due to the significant associations between silicosis and age, silicosis and occupation, and silicosis and cumulative service.

The incidence of TB in the subgroup tested for HIV is nearly double the rate found for the whole group. The association between HIV and TB is heavily negatively confounded by the inverse association between HIV and age. Once age, silicosis, occupation, and cumulative service have been controlled for, the data show a significant association between HIV infection and TB. This is likely to be underestimated for two reasons: firstly workers with HIV infection are increasingly likely to leave the mines as the infection progresses, and secondly this analysis could not take account of whether TB preceded HIV or not. The subgroup tested for HIV is a biased subsample of the main group but it constitutes nearly a third of the randomly selected study group of 5000 workers. Analysis of the subgroup tested for HIV shows no evidence of any positive associations between HIV and the other risk factors. This suggests that it is unlikely that any of the findings of the main study have come about as a result of confounding by HIV although this cannot be ruled out. On the other hand, it is likely that the effect of age has been underestimated as a result of negative confounding with HIV infection. The prevalence of HIV in the subgroup tested seems to have risen steeply up to 1994, and then levelled off. Whether this represents a slowing of the rate of infection, or changes in practices on testing cannot be ascertained from this trend. Informal reports suggest that increasing numbers of patients with sexually transmitted diseases refuse an HIV test, which could be the reason for an apparent reduction in the rate of infection.

\section{Conclusion}

This study has shown that as well as the excess risk of TB posed by HIV and silicosis, there is a highly significant association of incidence of TB with age and mining occupation. Neither of these results are likely to be due to any artefact in the data and the true effects of age and high risk occupation may well be larger than measured here. Any positive secular trend of incidence of TB may be a result of inadequacies in the data. The study provides no evidence of an association of the incidence of TB with cumulative service after adjusting for confounders, but it is likely that selection bias and exposure misclassification would have obscured any true association if one were to exist. The association of incidence of TB with silicosis is in line with what is to be expected from previous research, but the effect of silicosis on TB disappears once it is adjusted for factors with which it is strongly associated. This may well be due to poor ascertainment of silicosis in the data. Age is by far the strongest predictor of risk of tuberculosis with a relative risk between the highest and lowest age groups about 10 times the relative risk between occupation groups at high and low risk.

The results of this study indicate that there are groups of mineworkers who are at a higher risk of tuberculosis than others. To show this, profiles of the groups at lowest and highest risk would be as follows. The low risk worker is a 20 year old mineworker with less than two years service, working in a low dust job, and with no diagnosis of silicosis and no HIV infection. The annual incidence of tuberculosis in this group is just $<1 / 1000$ in 1995 . The high risk worker, by contrast, is a mineworker above the age of 55, with at least six years service, who has mostly worked in production jobs such as drilling, and who has been diagnosed as having silicosis. He would have been subject to an annual 
incidence of $\mathrm{TB}$ of $82.9 / 1000$. The two extremes represent a rate ratio of nearly 85 . Even without any radiological evidence of silicosis in the high risk worker, this rate ratio would still be about 50 . If the additional risk of tuberculosis posed by $\mathrm{HIV}^{21}$ to the high risk man is added, the annual incidence in such groups would be well above $10 \%$ even without any possible synergistic effect between HIV and silicosis on risk of TB.

This study confirms that TB rates among gold miners are high; prevention efforts should, therefore, include the whole workforce. This study also shows, however, that there is a profile for high risk within this population thus providing an opportunity for more targeted interventions. Interventions that should be considered for the high risk group are more frequent radiological screening, clinical screening, and chemoprophylaxis. This profile should be taken into account when existing screening policies in the gold mines are reviewed so that the most cost effective programmes can be implemented.

We thank Anglogold Health Services and the National Union of Mine Workers for having given support to this research. We thank Drs Brian Williams, Kevin DeCock, and Daan Mulder for critical suggestions on earlier drafts of this paper and Dr Beau Dees for facilitating access to the data of the Occupational Health Centre.

1 Van der Linde I. Stop the TB scourge. $S$ Afr Med $\mathcal{f}$ 1996;86:897-8

2 Mount Grace Symposium on TB in the mining industry. Johannesburg: Mine medical officers association newsletter. January 1996.

3 Chamber of Mines of South Africa. Figures quoted in the 1993 Annual Report of the Medical Division of Goldfields of South Africa Limited. Table 15. Johannesburg: Goldfields of South Africa, 1993.
4 Leon R, Davies A, Salomon M, Davies J. Report of the Commission of Inquiry into Safety and Health in the Mining industry March 1995. Pretoria: Government Printers, 1995 (Section 4.6.8.)

5 Leon R, Davies A, Salomon M, Davies J. Report of the Commission of Inquiry into Safety and Health in the Mining industry March 1995. Pretoria: Government Printers, 1995. (Section 4.5.15.)

6 White N. Health hazards in the mining industry: an overview report submitted to the Leon Commission of Enquiry into Health to the Commission of Enquiry into Health and Safety in to the Commission of Enquir
the Mining Industry, 1994 .

7 Cowie RL. The epidemiology of tuberculosis in gold miners with silicosis. Am $\mathcal{F}$ Respir Crit Care Med 1994;150:1460-2.

8 Balmes J. Silica exposure and tuberculosis: an old problem with some new twists. $\mathcal{F}$ Occup Med 1990;32:114-5

9 Cowie RL. The epidemiology of tuberculosis in gold miners with silicosis. Am 7 Repair Crit Care Med 1994;150:1460-2.

10 Cowie RL. Silicosis and tuberculosis. In: Coovadia HM Benatar SR, eds. A century off tuberculosis. Oxford: Oxford University Press, 1991.

11 Sluis-Cremer GK. Active pulmonary tuberculosis discovered at post-mortem examination of the lungs of black miners. Br $\mathcal{F}$ Dis Chest 1980;74:374

12 Murray J, Kielkowski D, Reid P. Occupational disease trends in black South African gold miners. Am $\mathcal{F}$ Respir Crit Care Med 1996;153:706-10.

13 Escreet BC, Cowie RL. Criteria for the diagnosis of pulmonary tuberculosis $S$ Afr Med F 1983;63:850-4

14 International Labour Office. Guidelines for the use of ILO international classification of radiographs of pneumoconiosis. international classification of radiogr

15 Clayton D, Hills M. Statistical models in epidemiology, Ch 6, 23. Oxford: Oxford University Press, 1993.

16 Stata Corporation. Wilcoxon rank-sum test as implemented in the nptrend command in Stata Statistics/Data analysis Version 4.0 for Windows. College Station, Texas: Stata Corporation, 1995.

17 Cowie RL, Langton ME, Becklake MR. Pulmonary tuberculosis in South African gold miners. Am Rev Respir Dis 1989;139:1086-9.

18 Cowie RL. Pulmonary tuberculosis in South African gold miners: determinants of relapse after treatment [MSc thesis]. miners: determinants of relapse after

19 Department of Health. Tuberculosis up-date in epidemiological comments. Pretoria: Department of Health, 1995;22:1:13

20 Strebel PM, Seager JR. Epidemiology of tuberculosis in South Africa. In: Coovadia HM, Benatar SR, eds. A century of tuberculosis. Oxford: Oxford University Press, 1991.

21 De Cock KM, Soro B, Coulibaly IM, Lucas SB. Tuberculosis and HIV in sub-Saharan Africa. $\mathcal{F A M A} 1$ 1992;268:1581-7. 